In print on Int. J. Non-Linear Mechanics manuscript No.

(will be inserted by the editor)

\title{
SO (3) INVARIANCE AND COVARIANCE IN MIXTURES OF SIMPLE BODIES
}

\author{
Paolo Maria Mariano \\ Dipartimento di Ingegneria Strutturale e Geotecnica, \\ Università di Roma "La Sapienza, \\ via Eudossiana 18, 00184 Roma (Italy), \\ e-mail: paolo.mariano@uniroma1.it \\ and Università del Molise, Campobasso (Italy). \\ The date of receipt and acceptance will be inserted by the editor
}

\begin{abstract}
We adapt to mixtures the procedure of invariance of external power under the action of $S O(3)$ to deduce balance equations. The two classical axioms about the growths of momentum and moment of momentum are derived with the help of a rule on the structure of the total power. We discuss also the covariance of the balance equations of each constituent and the nature of the constituent stress.
\end{abstract}

Key words Mixtures, invariance

1 Commentary to the theory of mixtures of simple bodies: the rôle of the invarance of the power.

\subsection{Preliminary remarks.}

Let $\mathcal{B}_{0}$ be the regular (in the sense of "fit") region of the three-dimensional Euclidean point space $\mathcal{E}^{3}$ occupied by the mixture in a reference place. We call part of $\mathcal{B}_{0}$ any regular subset $\mathfrak{b}$ of it and denote with $\mathbf{X}$ its generic point. The regular region $\mathcal{B}$ occupied by the mixture in the current place is obtained from $\mathcal{B}_{0}$ through a sufficiently smooth orientation preserving mapping $f$ such that $f\left(\mathcal{B}_{0}\right)=\mathcal{B}$; for any $\mathbf{x} \in \mathcal{B}$ we have then $\mathbf{x}=f(\mathbf{X})$. Motions are sufficiently smooth one-parameter mappings $f_{t}$, with $t \in\left[t_{0}, t_{1}\right]$ the time. The velocity in the spatial description is denoted with $\mathbf{v}$, while the acceleration with $\mathbf{a}=\partial_{t} \mathbf{v}+(\operatorname{grad} \mathbf{v}) \mathbf{v}$.

At the introduction of Lecture 5 of his "Rational Thermodynamics", Truesdell [1] describes the mixtures by assuming as a crucial point of view that all constituents occupy $\mathcal{B}$ simultaneously. Such an assumption is not 
true at a 'small' scale ${ }^{1}$ but it can be considered valid at the coarse scale that allows us to use the standard procedures of continuum mechanics. In this way, each point $\mathbf{x}$ of $\mathcal{B}$ has indeed the properties of all the constituents: with $\mathbf{x}_{\alpha}^{\prime}(\mathbf{x}, t)$ we indicate the velocity of the $\alpha$ th constituent at $\mathbf{x}$ (a spatial field), while with $\mathbf{x}_{\alpha}^{\prime \prime}$ the acceleration of the same constituent at $\mathbf{x}$ (the apex means here time derivative); the density of mass of the same constituent is $\rho_{\alpha}$, and $c_{\alpha}$ the concentration of mass, i.e. the ratio $c_{\alpha}=\frac{\rho_{\alpha}}{\rho}$, where $\rho=\sum_{\alpha} \rho_{\alpha}$. When we consider the mixture as a single body, the velocity $\mathbf{v}$ at $\mathbf{x}$ and $t$ is given by the sum of the velocities of each constituent weighed by the concentrations:

$$
\mathbf{v}=\sum_{\alpha} c_{\alpha} \mathbf{x}_{\alpha}^{\prime}
$$

Note that when all the constituents have the same velocity $\mathbf{x}^{\prime}=\mathbf{x}_{\alpha}^{\prime}$ for any $\alpha$, then $\mathbf{v}=\mathbf{x}^{\prime}$ because $\sum_{\alpha} c_{\alpha}=1$.

An external spatial observer calls rigid all the velocity fields $\mathbf{v}_{R}$ (the subscript R stands for 'rigid') of the form

$$
\mathbf{v}_{R}=\mathbf{c}(t)+\dot{\mathbf{q}} \times\left(\mathbf{x}-\mathbf{x}_{0}\right),
$$

where $\mathbf{c}(t)$ and $\dot{\mathbf{q}}(t)$ are translational and rotational velocities respectively. At a certain instant $t$, a spatial observer evaluates a velocity $\mathbf{v}$ (and $\mathbf{x}_{\alpha}^{\prime}$ for the $\alpha$ th constituent). If the observer changes its own state, new velocities $\mathbf{v}^{*}$ and $\mathbf{x}_{\alpha}^{\prime *}$ are measured and the values $\mathbf{v}$ and $\mathbf{v}^{*}\left(\right.$ or $\mathbf{x}_{\alpha}^{\prime}$ and $\mathbf{x}_{\alpha}^{\prime *}$ ) are related by the rules describing the change of state of the observer. We consider first changes of observer ruled by elements of the special orthogonal group $S O$ (3) (the observers differ trough a rigid body motion). We then have

$$
\mathbf{x}_{\alpha}^{\prime *}=\mathbf{x}_{\alpha}^{\prime}+\mathbf{c}(t)+\dot{\mathbf{q}} \times\left(\mathbf{x}-\mathbf{x}_{0}\right),
$$

which includes both Galilean (c constant in time and $\dot{\mathbf{q}}=\mathbf{0}$ ) and rotational $(\mathbf{c}=\mathbf{0}, \ddot{\mathbf{q}}=\mathbf{0})$ changes of observer.

Equation (3) implies that each observer sees all the constituents. This justify the choice of common translational and rotational velocities. Each observer is, in fact, a slicing of space-time. The simultaneous presence of constituents can be viewed as a simultaneous presence of slicings. With (3) we then presume that all slicings are isometrically 'deformed' with the same translational and rotational velocities.

\subsection{The main result.}

The picture of the simultaneous presence of all the constituents at each point $\mathbf{x}$ of $\mathcal{B}$ is enriched naturally when we try to represent the interactions within the mixture. The additional ingredient with respect to the mechanics of simple bodies is, in fact, the need to account for the interactions between

\footnotetext{
1 The physical meaning of the adjective 'small' changes for each type of mixture.
} 
each constituent and the others and to represent them in some way. Truesdell suggested three methaphysical principles for mixtures (see [1]): (i) the properties of the whole mixture are consequences of the ones of the constituents, (ii) each of them can be isolated from the rest provided that the interactions with the others are accounted for, (iii) the whole mixture behaves as a single body.

When these principles are applied to evaluate the balance of mass, taking into account (ii) we have

$$
\rho \breve{c}_{\alpha}=\partial_{t} \rho_{\alpha}+\operatorname{div}\left(\rho_{\alpha} \mathbf{x}_{\alpha}^{\prime}\right)
$$

for the $\alpha$ th constituent, where $\breve{c}_{\alpha}$ is the growth of mass of the same constituent. On the other hand, if the mixture is isolated, from the principles (i), (ii) and (iii) (chiefly from principle (iii)) we obtain by summation of (4) on $\alpha$

$$
\begin{gathered}
\dot{\rho}+\rho \operatorname{div} \mathbf{v}=0 \quad \text { in } \mathcal{B}, \\
\sum_{\alpha} \breve{c}_{\alpha}=0 .
\end{gathered}
$$

To deduce the balance equations for a mixture, we adapt here the procedure based on a request of invariance of the external power under changes of observers developed in [2]. This allows us to construct a tool useful for deriving models of mixtures of complex bodies. The basic outline of such a procedure is as follows:

- We write first the power developed by all external interactions on an arbitrary part of a certain constituent and include explicitly terms of interaction with the other constituents because, though all costituents are in the same place, each of them is "external" with respect to the others. A requirement of invariance of the power with respect to changes of observer ruled by (3) allows us to obtain integral (and - by localization - pointwise) balance equations of momentum and moment of momentum.

- We write also the external power on the same part of the mixture, considered globally in the spirit of principle (iii) and assume that it is the sum of the powers of the constituents calculated on the velocity of the whole mixture, for any choice of $\mathbf{v}$ (and we call this assumption "rule of the total power" for convenience).

The main result of this procedure is the proof that the growth of momentum on a given constituent due to the others is self-equilibrated, and the same holds for the growth of moment of momentum, while in the standard presentation of the theory these properties are assumed as axioms.

Let $\mathfrak{b}$ be any arbitrary part of the body, we write the power $\mathcal{P}_{\mathfrak{b}}^{\alpha}$ developed on the $\alpha$ th constituent occupying $\mathfrak{b}$ as

$$
\mathcal{P}_{\mathfrak{b}}^{\alpha}=\int_{\mathfrak{b}} \rho_{\alpha} \mathbf{b}_{\alpha} \cdot \mathbf{x}_{\alpha}^{\prime}+\int_{\partial \mathfrak{b}} \mathbf{T}_{\alpha} \mathbf{n} \cdot \mathbf{x}_{\alpha}^{\prime}+\int_{\mathfrak{b}} \rho \check{\mathbf{m}}_{\alpha} \cdot \mathbf{x}_{\alpha}^{\prime}+\int_{\mathfrak{b}} \rho \check{\boldsymbol{\mu}}_{\alpha} \cdot \operatorname{curl} \mathbf{x}_{\alpha}^{\prime},
$$


where $\mathbf{n}$ is the outward unit normal to the boundary $\partial \mathfrak{b}$ of $\mathfrak{b}, \mathbf{b}_{\alpha}$ the vector of body forces acting on the $\alpha$ th constituent, $\mathbf{T}_{\alpha}$ the Cauchy stress tensor on the same constituent, $\check{\mathbf{m}}_{\alpha}$ a vector representing the growth of momentum induced by the other constituents on the $\alpha$ th one, $\check{\boldsymbol{\mu}}_{\alpha}$ the analogous growth of moment of momentum. In the spirit of principle (ii), the last two integrals in (7) represent the power of the direct interactions between the $\alpha$ th constituent and the others.

The principle (iii) suggests also to write the power on a part $\mathfrak{b}$ occupied by the whole mixture as the power of a simple body. As a consequence, we write the standard relation

$$
\mathcal{P}_{\mathfrak{b}}=\int_{\mathfrak{b}} \rho \mathbf{b} \cdot \mathbf{v}+\int_{\partial \mathfrak{b}} \mathbf{T n} \cdot \mathbf{v},
$$

where $\mathbf{b}$ is the vector of the total bulk interactions and $\mathbf{T}$ the total Cauchy stress.

At each $\mathbf{x}, \mathbf{b}$ and $\mathbf{b}_{\alpha}$ and $\mathbf{T}$ and $\mathbf{T}_{\alpha}$ are related (see [1]) by

$$
\begin{gathered}
\mathbf{b}=\sum_{\alpha} c_{\alpha} \mathbf{b}_{\alpha} \\
\mathbf{T}=\sum_{\alpha} \mathbf{T}_{\alpha} .
\end{gathered}
$$

Note that the bulk measures of interaction $\mathbf{b}$ and $\mathbf{b}_{\alpha}$ are comprehensive of inertial actions and we consider valid the additive decomposition into the inertial (in) and non-inertial $(n i)$ components, namely

$$
\begin{gathered}
\mathbf{b}_{\alpha}=\mathbf{b}_{\alpha}^{n i}+\mathbf{b}_{\alpha}^{i n}, \\
\mathbf{b}=\mathbf{b}^{n i}+\mathbf{b}^{i n} .
\end{gathered}
$$

Theorem 1 In the theory of mixtures of simple bodies, the two groups of assertions listed in what follows are equivalent.

First group.

- $\mathcal{P}_{\mathfrak{b}}^{\alpha}$ is invariant under classical changes of observer ruled by (3), i.e.

$$
\mathcal{P}_{\mathfrak{b}}^{\alpha}\left(\mathbf{x}_{\alpha}^{\prime *}\right)=\mathcal{P}_{\mathfrak{b}}^{\alpha}\left(\mathbf{x}_{\alpha}^{\prime}\right)
$$

for any choice of $\mathfrak{b}, \mathbf{c}(t)$ and $\dot{\mathbf{q}}(t)$.

- The following rule of the kinetic energy and the power of inertial forces holds:

$$
\begin{gathered}
\frac{d}{d t} \int_{\mathfrak{b}} \frac{1}{2} \rho_{\alpha} \mathbf{x}_{\alpha}^{\prime} \cdot \mathbf{x}_{\alpha}^{\prime}-\frac{1}{2} \int_{\partial \mathfrak{b}} \rho_{\alpha} \mathbf{x}_{\alpha}^{\prime} \cdot \mathbf{x}_{\alpha}^{\prime}\left(\mathbf{x}_{\alpha}^{\prime} \cdot \mathbf{n}\right)+ \\
\quad+\frac{1}{2} \int_{\mathfrak{b}} \rho \breve{c}_{\alpha} \mathbf{x}_{\alpha}^{\prime} \cdot \mathbf{x}_{\alpha}^{\prime}+\int_{\mathfrak{b}} \rho_{\alpha} \mathbf{b}_{\alpha}^{i n} \cdot \mathbf{x}_{\alpha}^{\prime}=0
\end{gathered}
$$

for any $\mathfrak{b}$. 
- The rule of the total power holds:

$$
\mathcal{P}_{\mathfrak{b}}(\mathbf{v})=\sum_{\alpha} \mathcal{P}_{\mathfrak{b}}^{\alpha}(\mathbf{v})
$$

for any choice of $\mathbf{v}$.

Second group.

- For each constituent, the following equations of balance of momentum and moment of momentum hold:

$$
\begin{gathered}
\rho_{\alpha} \mathbf{b}_{\alpha}^{n i}+\operatorname{div} \mathbf{T}_{\alpha}+\rho \check{\mathbf{m}}_{\alpha}=\rho c_{\alpha} \mathbf{x}_{\alpha}^{\prime \prime}+\rho \breve{c}_{\alpha} \mathbf{x}_{\alpha}^{\prime}, \\
\mathbf{T}_{\alpha}-\mathbf{T}_{\alpha}^{T}=-\rho \check{\boldsymbol{\mu}}_{\alpha} \times
\end{gathered}
$$

- The growths of momentum due to the interactions between constituents are pointwise self-equilibrated:

$$
\sum_{\alpha} \check{\mathbf{m}}_{\alpha}=0
$$

- The growths of moment of momentum due to the interactions between constituents are pointwise self-equilibrated:

$$
\sum_{\alpha} \check{\boldsymbol{\mu}}_{\alpha}=0
$$

- For the whole mixture, the following equations of balance of momentum and moment of momentum hold:

$$
\begin{gathered}
\rho \mathbf{b}^{n i}+\operatorname{div}\left(\mathbf{T}+\sum_{\alpha} c_{\boldsymbol{\alpha}} \mathbf{u}_{\alpha} \otimes \mathbf{u}_{\alpha}\right)=\rho \mathbf{a}, \\
s k w \mathbf{T}=\mathbf{0}
\end{gathered}
$$

where $\mathbf{u}_{\alpha}=\mathbf{x}_{\alpha}^{\prime}-\mathbf{v}$ is the velocity of diffusion of the $\alpha$ th constituent.

In (20), the term $\sum_{\boldsymbol{\alpha}} c_{\boldsymbol{\alpha}} \mathbf{u}_{\alpha} \otimes \mathbf{u}_{\alpha}$ accounts for the diffusive motion of the constituents of the mixture. Analogous terms are present in the kinetic theory of gases. 


\subsection{Proof of Theorem 1.}

We prove here only that the second group of assertions follows from the first group, because the converse is rather immediate.

First we consider the power $\mathcal{P}_{\mathfrak{b}}^{\alpha}$ and realize that the request of invariance (13) is satisfied if and only if

$$
\begin{gathered}
\mathbf{c} \cdot\left(\int_{\mathfrak{b}} \rho_{\alpha} \mathbf{b}_{\alpha}+\int_{\partial \mathfrak{b}} \mathbf{T}_{\alpha} \mathbf{n}+\int_{\mathfrak{b}} \rho \check{\mathbf{m}}_{\alpha}\right)+ \\
+\dot{\mathbf{q}} \cdot\left(\int_{\mathfrak{b}}\left(\mathbf{x}-\mathbf{x}_{0}\right) \times \rho_{\alpha} \mathbf{b}_{\alpha}+\int_{\partial \mathfrak{b}}\left(\mathbf{x}-\mathbf{x}_{0}\right) \times \mathbf{T}_{\alpha} \mathbf{n}\right)+ \\
+\dot{\mathbf{q}} \cdot\left(\int_{\mathfrak{b}}\left(\mathbf{x}-\mathbf{x}_{0}\right) \times \rho \check{\mathbf{m}}_{\alpha}+\int_{\mathfrak{b}} \rho \check{\boldsymbol{\mu}}_{\alpha}\right)=0,
\end{gathered}
$$

for any choice of $\mathbf{c}$ and $\dot{\mathbf{q}}$, whose arbitraryness implies the integral balances of forces and couples for the $\alpha$ th constituent:

$$
\begin{gathered}
\int_{\mathfrak{b}} \rho_{\alpha} \mathbf{b}_{\alpha}+\int_{\partial \mathfrak{b}} \mathbf{T}_{\alpha} \mathbf{n}+\int_{\mathfrak{b}} \rho \check{\mathbf{m}}_{\alpha}=0 \\
\int_{\mathfrak{b}}\left(\mathbf{x}-\mathbf{x}_{0}\right) \times \rho_{\alpha} \mathbf{b}_{\alpha}+\int_{\partial \mathfrak{b}}\left(\mathbf{x}-\mathbf{x}_{0}\right) \times \mathbf{T}_{\alpha} \mathbf{n}+\int_{\mathfrak{b}}\left(\mathbf{x}-\mathbf{x}_{0}\right) \times \rho \check{\mathbf{m}}_{\alpha}+\int_{\mathfrak{b}} \rho \check{\boldsymbol{\mu}}_{\alpha}=0 .
\end{gathered}
$$

The arbitraryness of $\mathfrak{b}$ and the use of the Gauss theorem allow us to obtain from (23) the pointwise balance

$$
\rho_{\alpha} \mathbf{b}_{\alpha}+\operatorname{div} \mathbf{T}_{\alpha}+\rho \check{\mathbf{m}}_{\alpha}=0,
$$

while equation (17) follows from (24) with the additional use of (25).

A standard transport theorem and the use of the balance of mass (4) allow us to calculate the time derivative of the first integral in (14): we then have

$$
\frac{1}{2} \frac{d}{d t} \int_{\mathfrak{b}} \rho_{\alpha} \mathbf{x}_{\alpha}^{\prime} \cdot \mathbf{x}_{\alpha}^{\prime}=\int_{\mathfrak{b}} \rho_{\alpha} \mathbf{x}_{\alpha}^{\prime \prime} \cdot \mathbf{x}_{\alpha}^{\prime}+\frac{1}{2} \int_{\partial \mathfrak{b}} \frac{1}{2} \rho_{\alpha} \mathbf{x}_{\alpha}^{\prime} \cdot \mathbf{x}_{\alpha}^{\prime}\left(\mathbf{x}_{\alpha}^{\prime} \cdot \mathbf{n}\right)+\int_{\mathfrak{b}} \frac{1}{2} \rho \breve{c}_{\alpha} \mathbf{x}_{\alpha}^{\prime} \cdot \mathbf{x}_{\alpha}^{\prime}
$$

By inserting (26) in (14) we obtain

$$
\int_{\mathfrak{b}}\left(\rho_{\alpha} \mathbf{x}_{\alpha}^{\prime \prime}+\rho \breve{c}_{\alpha} \mathbf{x}_{\alpha}^{\prime}+\rho_{\alpha} \mathbf{b}_{\alpha}^{i n}\right) \cdot \mathbf{x}_{\alpha}^{\prime}=0
$$

Even in this case both $\mathfrak{b}$ and $\mathbf{x}_{\alpha}^{\prime}$ are arbitrary, then

$$
\rho_{\alpha} \mathbf{b}_{\alpha}^{i n}=-\rho_{\alpha} \mathbf{x}_{\alpha}^{\prime \prime}-\rho \breve{c}_{\alpha} \mathbf{x}_{\alpha}^{\prime}
$$

By substituting (28) and (11) in (25), the pointwise balance of momentum of the $\alpha$ th constituent (16) follows. 
Since the rule of the total power holds for any choice of $\mathbf{v}$, it holds for rigid velocity fields of the form (2). As a consequence, from (15) we obtain

$$
\begin{aligned}
\mathbf{c} \cdot\left(\int_{\mathfrak{b}} \rho \mathbf{b}+\right. & \left.\int_{\partial \mathfrak{b}} \mathbf{T n}\right)+\dot{\mathbf{q}} \cdot\left(\int_{\mathfrak{b}}\left(\mathbf{x}-\mathbf{x}_{0}\right) \times \rho \mathbf{b}+\int_{\partial \mathfrak{b}}\left(\mathbf{x}-\mathbf{x}_{0}\right) \times \mathbf{T n}\right)= \\
= & \mathbf{c} \cdot\left(\int_{\mathfrak{b}} \sum_{\alpha}\left(\rho_{\alpha} \mathbf{b}_{\alpha}+\rho \check{\mathbf{m}}_{\alpha}\right)+\int_{\partial \mathfrak{b}} \sum_{\alpha} \mathbf{T}_{\alpha} \mathbf{n}\right)+ \\
& +\dot{\mathbf{q}} \cdot\left(\int_{\mathfrak{b}} \sum_{\alpha}\left(\mathbf{x}-\mathbf{x}_{0}\right) \times\left(\rho_{\alpha} \mathbf{b}_{\alpha}+\rho \check{\mathbf{m}}_{\alpha}\right)\right)+ \\
& +\dot{\mathbf{q}} \cdot\left(\int_{\partial \mathfrak{b}} \sum_{\alpha}\left(\mathbf{x}-\mathbf{x}_{0}\right) \times \mathbf{T}_{\alpha} \mathbf{n}+\int_{\mathfrak{b}} \sum_{\alpha} \rho \check{\boldsymbol{\mu}}_{\alpha}\right)
\end{aligned}
$$

i.e.

$$
\begin{gathered}
\int_{\mathfrak{b}} \rho \mathbf{b}+\int_{\partial \mathfrak{b}} \mathbf{T n}=\int_{\mathfrak{b}} \sum_{\alpha}\left(\rho_{\alpha} \mathbf{b}_{\alpha}+\rho \check{\mathbf{m}}_{\alpha}\right)+\int_{\partial \mathfrak{b}} \sum_{\alpha} \mathbf{T}_{\alpha} \mathbf{n} \\
\int_{\mathfrak{b}}\left(\mathbf{x}-\mathbf{x}_{0}\right) \times \rho \mathbf{b}+\int_{\partial \mathfrak{b}}\left(\mathbf{x}-\mathbf{x}_{0}\right) \times \mathbf{T} \mathbf{n}= \\
=\int_{\mathfrak{b}} \sum_{\alpha}\left(\mathbf{x}-\mathbf{x}_{0}\right) \times\left(\rho_{\alpha} \mathbf{b}_{\alpha}+\rho \check{\mathbf{m}}_{\alpha}\right)+\int_{\partial \mathfrak{b}} \sum_{\alpha}\left(\mathbf{x}-\mathbf{x}_{0}\right) \times \mathbf{T}_{\alpha} \mathbf{n}+\int_{\mathfrak{b}} \sum_{\alpha} \rho \check{\boldsymbol{\mu}}_{\alpha} .
\end{gathered}
$$

By inserting (9) and (10) in (30) and (31), we obtain (18) and (19) thanks to the arbitraryness of $\mathfrak{b}$.

The balance equations (20) and (21) of the whole mixture follow by summing over $\alpha$ equations (16) and (17); we make use of (18) and (19) and take into account that for any spatial field $\lambda(\mathbf{x}, t)=\sum_{\alpha} c_{\alpha} \lambda_{\alpha}(\mathbf{x}, t)$ we have (see [1])

$$
\dot{\lambda}=\sum_{\alpha} c_{\alpha} \lambda_{\alpha}^{\prime}-\rho^{-1} d i v \sum_{\alpha} c_{\alpha} \lambda_{\alpha} \mathbf{u}_{\alpha}+\sum_{\alpha} \breve{c}_{\alpha} \lambda_{\alpha}
$$

Remark (about boundary conditions). The problem of assigning boundary conditions in mixtures is in general rather delicate (and crucial) because we know the total boundary traction $\mathbf{T n}=\mathbf{t}$ and do not know the constituent traction $\mathbf{T}_{\alpha} \mathbf{n}=\mathbf{t}_{\alpha}$. The question has been discussed in detail by Rajagopal and co-workers (here we do not tackle the problem) and the related results (together with comparisons with the proposals in the existing literature) can be found in [3] (see also $[4,5]$ ). 


\section{A theorem of the kinetic energy and the power of inertial forces for the whole mixture.}

In the present Section we reverse the point of view followed so far: we do not consider the single constituent but account for the whole mixture by connecting (8) with (20) through the use of an appropriate theorem of the kinetic energy and the power of inertial forces.

Basically, we consider the term $\operatorname{div} \sum_{\boldsymbol{\alpha}} c_{\boldsymbol{\alpha}} \mathbf{u}_{\alpha} \otimes \mathbf{u}_{\alpha}$ of inertial nature and associate it with $\mathbf{b}^{i n}$. This interpretation is not standard. The decomposition (11) is in general adopted to separate the objective part of the bulk interactions (namely $\mathbf{b}^{n i}$ ) from the rest: the inertial terms are, in fact, not objective. Here the term $\mathbf{u}_{\alpha} \otimes \mathbf{u}_{\alpha}$ is objective, so we consider $\mathbf{b}^{i n}$ endowed with an objective part.

The analogous expression of (3) for $\mathbf{v}$ is

$$
\mathbf{v}^{*}=\mathbf{v}+\mathbf{c}(t)+\dot{\mathbf{q}} \times\left(\mathbf{x}-\mathbf{x}_{0}\right) .
$$

The values $\mathbf{v}$ and $\mathbf{v}^{*}$ are the ones measured respectively before and after "rigid" changes of observer ruled by $S O(3)$. If we require the invariance of $\mathcal{P}_{\mathfrak{b}}$ in (8) under (33), we obtain the standard pointwise balance

$$
\rho \mathbf{b}+\operatorname{div} \mathbf{T}=\mathbf{0},
$$

in which (11) applies. Taking into account (10), the compatibility of (34) with (20) is assured if we postulate for the whole mixture that

$$
\begin{aligned}
& \frac{d}{d t} \int_{\mathfrak{b}} \frac{1}{2} \rho \mathbf{v} \cdot \mathbf{v}+\int_{\partial \mathfrak{b}} \sum_{\alpha} c_{\alpha} \mathbf{u}_{\alpha} \cdot \mathbf{v}\left(\mathbf{u}_{\alpha} \cdot \mathbf{n}\right)+ \\
& +\int_{\mathfrak{b}} \sum_{\boldsymbol{\alpha}} c_{\boldsymbol{\alpha}} \mathbf{u}_{\alpha} \otimes \mathbf{u}_{\alpha} \cdot \operatorname{grad} \mathbf{v}+\int_{\mathfrak{b}} \rho \mathbf{b}^{i n} \cdot \mathbf{v}=0
\end{aligned}
$$

for any fixed control volume $\mathfrak{b}$ in $\mathcal{B}$.

- Since $\mathfrak{b}$ is fixed, we interpret

$$
\int_{\partial \mathfrak{b}} \sum_{\alpha} c_{\alpha} \mathbf{u}_{\alpha} \cdot \mathbf{v}\left(\mathbf{u}_{\alpha} \cdot \mathbf{n}\right)
$$

as the total flow of kinetic energy through the boundary $\partial \mathfrak{b}$ due to the relative motion of the constituents with respect to $\mathfrak{b}$.

- Moreover, the term

$$
\int_{\mathfrak{b}} \sum_{\alpha} c_{\boldsymbol{\alpha}} \mathbf{u}_{\alpha} \otimes \mathbf{u}_{\alpha} \cdot \operatorname{grad} \mathbf{v}
$$

is interpreted as a production of kinetic energy due to the cooperation of the non-uniformity of the velocity of the whole mixture and the local relative agitation of the constituents. 


\section{The first and the second principle of thermodynamics}

For the first principle in $\mathfrak{b}$, we simply add a "source" term to the standard expression. This term is due to the simultaneous presence of all constituents in $\mathfrak{b}$, which is assumed to be fixed. By indicating with $\varepsilon_{\alpha}$ the density of internal energy of the $\alpha$ th constituent and with $E_{\mathfrak{b}}^{\alpha}$ the supply of energy in $\mathfrak{b}$ due to the other constituents, we write

$$
\frac{d}{d t} \int_{\mathfrak{b}} \rho_{\alpha} \varepsilon_{\alpha}-E_{\mathfrak{b}}^{\alpha}-\mathcal{P}_{\mathfrak{b}}^{\alpha}\left(\mathbf{x}_{\alpha}^{\prime}\right)-\int_{\partial \mathfrak{b}} \mathbf{q}_{\alpha} \cdot \mathbf{n}-\int_{\mathfrak{b}} \rho_{\alpha} r_{\alpha}=0,
$$

where $\mathbf{q}_{\alpha}$ is the density of heat flux through the $\alpha$ th constituent and $r_{\alpha}$ the relevant heat source. For $E_{\mathfrak{b}}^{\alpha}$, the simplest choice is

$$
E_{\mathfrak{b}}^{\alpha}=\int_{\mathfrak{b}} \rho \breve{\varepsilon}_{\alpha}
$$

where $\breve{\varepsilon}_{\alpha}$ is the growth of internal energy induced on the $\alpha$ th constituent by the other ones. By inserting (7) and (39) in (38) and taking into account the arbitraryness of $\mathfrak{b}$, we obtain the pointwise balance of energy

$$
\rho_{\alpha} \varepsilon_{\alpha}^{\prime}+\rho \breve{c}_{\alpha} \varepsilon_{\alpha}-\rho \breve{\varepsilon}_{\alpha}-\mathbf{T}_{\alpha} \cdot \operatorname{symgrad} \mathbf{x}_{\alpha}^{\prime}-\operatorname{div} \mathbf{q}_{\alpha}-\rho_{\alpha} r_{\alpha}=0,
$$

which has been suggested in [6] (sym extracts the symmetric part of its argument).

To render (38) compatible with the pointwise balance of energy suggested by Truesdell (see [1] and [3]), we should assume for $E_{\mathfrak{b}}^{\alpha}$ the following expression:

$$
E_{\mathfrak{b}}^{\alpha}=\int_{\mathfrak{b}}\left(\rho \breve{\varepsilon}_{\alpha}+\frac{1}{2} \rho \breve{c}_{\alpha} \mathbf{x}_{\alpha}^{\prime} \cdot \mathbf{x}_{\alpha}^{\prime}-\rho \check{\mathbf{m}}_{\alpha} \cdot \mathbf{x}_{\alpha}^{\prime}-\rho \check{\boldsymbol{\mu}}_{\alpha} \cdot \operatorname{curl} \mathbf{x}_{\alpha}^{\prime}\right) .
$$

In writing (41) we would presume that, given a certain constituent $\alpha$ in $\mathfrak{b}$, the others there have a twofold nature: from one hand they are external to the $\alpha$ th one (from which the presence of the interaction terms in (7)), while from the other hand, since all the constituents occupy $\mathfrak{b}$, the interaction terms in (7) contribute also to the supply of the internal energy. In other words, the supply of power furnished by the other constituents would be also interpreted as a supply of internal energy. In this way, by using (7), (41) and developing the time derivative in (38), the arbitraryness of $\mathfrak{b}$ implies the pointwise balance

$$
\begin{gathered}
\rho_{\alpha} \varepsilon_{\alpha}^{\prime}+\rho \breve{c}_{\alpha} \varepsilon_{\alpha}-\rho \breve{\varepsilon}_{\alpha}+\rho \check{\mathbf{m}}_{\alpha} \cdot \mathbf{x}_{\alpha}^{\prime}-\mathbf{T}_{\alpha} \cdot \operatorname{grad} \mathbf{x}_{\alpha}^{\prime}-\frac{1}{2} \rho \breve{c}_{\alpha} \mathbf{x}_{\alpha}^{\prime} \cdot \mathbf{x}_{\alpha}^{\prime}- \\
-\operatorname{div} \mathbf{q}_{\alpha}-\rho_{\alpha} r_{\alpha}=0
\end{gathered}
$$

which is the one in [1] and [3]. 
The first principle for the whole mixture is deduced by summation over $\alpha$, as a consequence of principle (iii). In developing the summation, following [1], one assumes as axiom that

$$
\sum_{\alpha} \breve{\varepsilon}_{\alpha}=0
$$

For the second principle, we have the standard expression

$$
\frac{d}{d t} \int_{\mathfrak{b}} \rho_{\alpha} \eta_{\alpha} \geq \int_{\mathfrak{b}} \rho \breve{\eta}_{\alpha}+\int_{\partial \mathfrak{b}} \mathbf{h}_{\alpha}-\int_{\mathfrak{b}} \rho_{\alpha} s_{\alpha},
$$

where $\eta_{\alpha}$ is the density of entropy in the $\alpha$ th constituent, $\breve{\eta}_{\alpha}$ the growth of entropy induced by the other constituents, $\mathbf{h}_{\alpha}$ and $s_{\alpha}$ entropy flux and source respectively. The pointwise thermodynamic relations derived and the integral ones may be exploited by making use of basic results in hermodynamics of mixtures [3, 7-9].

\section{Covariance}

Each observer is a special representation of space and time. Covariance means invariance with respect to changes of observer ruled by sufficiently smooth time-parametrized families of diffeomorphisms $\zeta_{t}: \mathcal{E}^{3} \rightarrow \mathcal{E}^{3}$ such that $\zeta_{0}=i d$ (id means identity).

In particular, we consider $n$ syncronous copies of the same slicing ( $n$ is the number of constituents); each copy is occupied by one constituent. To analize the covariance of the balance equations of the $\alpha$ th constituent, we consider an arbitrary $\zeta_{t}^{\alpha}$ acting only on the slicing of the relevant constituent. Through $\zeta^{\alpha}$ we deform the space, push-forward the fields defined on it, and denote with the subscript \# the images after $\zeta^{\alpha}$. We denote with $\mathbf{w}_{\alpha}$ the derivative $\dot{\zeta}_{0}^{\alpha}$. If we write $\mathfrak{E}(\mathfrak{b})=0$ for the balance (38), covariance is the requirement that

$$
\left.\mathfrak{E}_{\#}\left(\zeta_{t}^{\alpha}(\mathfrak{b})\right)\right|_{t=0}-\mathfrak{E}(\mathfrak{b})=0,
$$

for any $\zeta^{\alpha}$. For the sake of simplicity we assume that the two observers have the same time scale.

In principle, we presume that each constituent has his peculiar metric $\mathrm{g}_{\alpha}$.

Proposition 1 Let the explicit representation of $E_{\mathfrak{b}}^{\alpha}$ be given by (39). (a) The balance equations (16) and (17) are covariant. (b) The symmetric part of $\mathbf{T}_{\alpha}$ in $\mathfrak{b}$ satisfies the Doyle-Ericksen formula

$$
\operatorname{sym} \mathbf{T}_{\alpha}=2 \rho_{\alpha} \frac{\partial \varepsilon_{\alpha}}{\partial \mathbf{g}_{\alpha}} .
$$




\subsection{Proof of Proposition 1}

For the proof we follow the program discussed in [10] for the covariance of balance equations of simple bodies. Since $\mathbf{x}_{\# \alpha}=\zeta^{\alpha}(\mathbf{x}, t)$, its time derivative evaluated at $t=0$ gives rise to the following representation for the peculiar velocity of the $\alpha$ th constituent after $\zeta^{\alpha}$ :

$$
\mathbf{x}_{\# \alpha}^{\prime}=\mathbf{x}_{\alpha}^{\prime}+\mathbf{w}_{\alpha} .
$$

Under the action of $\zeta^{\alpha}$, the mass density $\rho_{\alpha}$ and the heat source $r_{\alpha}$ defined on $\mathcal{B}$ remain unchanged, while vector fields are pushed forward by the tangent map $T \zeta_{t}^{\alpha}$ which is the identity at $t=0$. A different case occurs for the internal energy which changes "tensorially" (see [10]). Under the suggestions of [10], we define

$$
\varepsilon_{\# \alpha}\left(\mathbf{x}_{\#}, t\right)=\varepsilon_{\alpha}\left(\mathbf{x}, t, \zeta_{t}^{\alpha *} \circ \mathbf{g}_{\alpha}\right),
$$

where $\zeta_{t}^{\alpha *}$ denotes pull-back. The meaning of (48) is discussed in [10] together with the need to parametrize $\varepsilon$ with the metric. Here, we specialize that point of view to the $\alpha$ th constituent and presume that in principle each constituent has its peculiar metric. In principle $\varepsilon_{\alpha}$ depends on the $\mathbf{g}_{\beta}$ 's, $\beta \neq \alpha$, characterizing the other constituents. However, the $\mathbf{g}_{\beta}$ 's live on slicings different from the $\alpha$ th one, then they are not pushed forward by $\zeta^{\alpha}$ and in (511) play the rôle of parameters. By chain rule, from (48) we have

$$
\dot{\varepsilon}_{\# \alpha}=\dot{\varepsilon}_{\alpha}+\frac{\partial \varepsilon_{\alpha}}{\partial \mathbf{g}_{\alpha}} \cdot L_{\mathbf{w}_{\alpha}} \mathbf{g}_{\alpha}
$$

where $L_{\mathbf{w}_{\alpha}}$ is the hautonomous Lie derivative of $\mathbf{g}_{\alpha}$ following $\mathbf{w}_{\alpha}$. First we write

$$
\begin{gathered}
\mathfrak{E}_{\#}\left(\zeta_{t}^{\alpha}(\mathfrak{b})\right)=\frac{d}{d t} \int_{\zeta_{t}(\mathfrak{b})} \rho_{\# \alpha} \varepsilon_{\# \alpha}-\int_{\zeta_{t}(\mathfrak{b})} \rho_{\#} \breve{\varepsilon}_{\# \alpha}-\int_{\zeta_{t}(\mathfrak{b})} \rho_{\# \alpha} \mathbf{b}_{\# \alpha} \cdot \mathbf{x}_{\# \alpha}^{\prime}- \\
-\int_{\partial \zeta_{t}(\mathfrak{b})} \mathbf{T}_{\# \alpha} \mathbf{n}_{\#} \cdot \mathbf{x}_{\# \alpha}^{\prime}-\int_{\zeta_{t}(\mathfrak{b})} \rho_{\#} \check{\mathbf{m}}_{\# \alpha} \cdot \mathbf{x}_{\# \alpha}^{\prime}-\int_{\zeta_{t}(\mathfrak{b})} \rho_{\#} \check{\boldsymbol{\mu}}_{\# \alpha} \cdot \operatorname{curl} \mathbf{x}_{\# \alpha}^{\prime}- \\
-\int_{\partial \zeta_{t}(\mathfrak{b})} \mathbf{q}_{\# \alpha} \cdot \mathbf{n}_{\#}-\int_{\zeta_{t}(\mathfrak{b})} \rho_{\# \alpha} r_{\# \alpha} .
\end{gathered}
$$

By using Gauss theorem and taking into account that 2 symgrad $\mathbf{x}_{\alpha}^{\prime}=$ $L_{\mathbf{w}_{\alpha}} \mathbf{g}_{\alpha}$, and that $\breve{\varepsilon}_{\# \alpha}=\breve{\varepsilon}_{\alpha}$ (the analogous of (501), (45) becomes

$$
\begin{gathered}
\left.\mathfrak{E}_{\#}\left(\zeta_{t}^{\alpha}(\mathfrak{b})\right)\right|_{t=0}-\mathfrak{E}(\mathfrak{b})=\int_{\mathfrak{b}}\left(\rho_{\alpha} \frac{\partial \varepsilon_{\alpha}}{\partial \mathbf{g}_{\alpha}}-\frac{1}{2} \operatorname{sym} \mathbf{T}_{\alpha}\right) \cdot L_{\mathbf{w}_{\alpha}} \mathbf{g}_{\alpha}- \\
-\int_{\mathfrak{b}}\left(\rho_{\alpha} \mathbf{b}_{\alpha}+\operatorname{div} \mathbf{T}_{\alpha}+\rho \check{\mathbf{m}}_{\alpha}\right) \cdot \mathbf{w}-\int_{\mathfrak{b}}\left(\operatorname{skw} \mathbf{T}_{\alpha}+\rho \mathbf{e} \check{\boldsymbol{\mu}}_{\alpha}\right) \cdot \operatorname{skwgrad} \mathbf{w}_{\alpha}=0,
\end{gathered}
$$


where e is the Ricci's alternating symbol and skw extracts the skew-symmetric part of its argument. The arbitraryness of $\mathbf{w}_{\alpha}$ implies that the terms in parentheses must vanish. We then obtain the balance equations from the last two terms (the last but one coincides with (25)). In addition, we have also (46).

Remark. When there is no exchange of couples between constituents $\left(\check{\boldsymbol{\mu}}_{\alpha}=\mathbf{0}\right.$ ), and $\varepsilon_{\alpha}$ doen not depend on the $\mathbf{g}_{\beta}$ 's, the Cauchy stress $\mathbf{T}_{\alpha}$ on $\mathfrak{b}$ measures contact interactions exerted only by the rest of the $\alpha$ th constituent. This result is has a link with the interpretation given by Williams of mixture theory in $[11,12]$, in particular with his "classical interpretation" when the mixture is not diffusive. In this case, the constituent stress is a measure of contact interactions between elements of the algebra of parts of the costituent itself, as in the case of simple bodies: an interpretation close to principle (ii).

Acknowledgement. I owe gratitude to Gianfranco Capriz for profound discussions.

\section{References}

1. Truesdell, C. A., Rational thermodynamics, Springer Verlag, Berlin, 1984.

2. Noll, W. (1973), Lectures on the foundations of continuum mechanics and thermodynamics, Arch. Rational Mech. Anal., 52, 62-92.

3. Rajagopal, K. R. and Tao, L., Mechanics of mixtures, World Scientific, Singapore, 1995.

4. Tao, L. and Rajagopal, K. R. (1994), On boundary conditions in mixture theory, in Recent Advances in Elasticity and Viscoelasticity, K. R. Rajagopal ed., World Scientific Publishing, Singapore.

5. Rajagopal, K. R., Wineman, A. S. and Gandhi, M. V. (1986), On boundary conditions for a certain class of problems in mixture theory, Int. $J$. Engng Sci., 29, 1453-1463.

6. Craine, R. E., Green, A. E., Naghdi, P. M. (1970), A mixture of viscous elastic materials with different constituent temperatures, Quarterly J. Mech. Appl. Math., 23, 171-184.

7. Capriz, G. (1990), Alcune osservazioni sulla termomeccanica delle miscele binarie, Riv. Mat. Univ. Parma, 16, 63-71.

8. Müller, I. (1967), A thermodynamic theory of mixtures of fluids, Arch. Rational Mech. Anal., 28, 1-39.

9. Müller, I., Thermodynamics, Pitman, 1985.

10. Marsden, J. E. and Hughes, T. J. R., Mathematical foundations of elasticity, Prentice-Hall inc., Englewood Cliffs, 1983.

11. Williams, W. O. (1979), On stresses in mixtures, Arch. Rational Mech. Anal., 70, 251-260.

12. Williams, W. O. (1973), On the theory of mixtures, Arch. Rational Mech. Anal., 51, 239-260. 